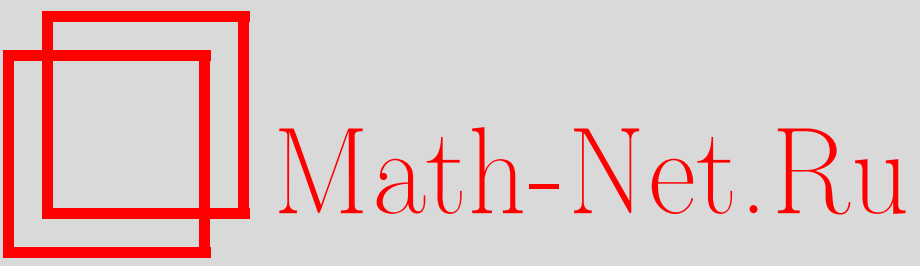

А. П. Форди, Т. Б. Гюрель, Новая конструкция рекурсионных операторов для систем гидродинамического типа, ТМФ, 2000, том 122, номер 1, 37-49

DOI: https://doi.org/10.4213/tmf553

Использование Общероссийского математического портала Math-Net.Ru подразумевает, что вы прочитали и согласны с пользовательским соглашением

http://www.mathnet.ru/rus/agreement

Параметры загрузки:

IP : 54.172 .240 .79

26 апреля 2023 г., $17: 30: 58$ 
ТЕОРЕТИЧЕСКАЯ

И МАТЕМАТИЧЕСКАЯ

ФИЗИКА

Том 122, № 1

январь, 2000

(C) 2000 r.

А. П. Форди* , Т. Б. Гюрель ${ }^{*}, \dagger$

\section{НОВАЯ КОНСТРУКЦИЯ РЕКУРСИОННЫХ ОПЕРАТОРОВ ДЛЯ СИСТЕМ ГИДРОДИНАМИЧЕСКОГО ТИПА}

Рассмотрен некоторый класс двумерных систем гидродинамического типа, который содержит все известные нам примеры и который можно связать с классом линейных волновых уравнений, обладающих алгеброй лестничных операторов. Подход применяется для простого построения рекурсионных операторов, которые не всегда совпадают с операторами Шефотеля-Тешукова.

\section{1. ВВЕДЕНИЕ}

В данной статье рассмотрены системы гидродинамического типа в смысле Дубровина и Новикова (см. обзоры $[1,2])$ и, в частности, рекурсионные операторы, введенные и обсуждаемые в [3, 4]. Шефьель показал (см. обзор [5]), что общий рекурсионньй оператор, ассоциированный с общей двумерной диагональной системой гидродинамического типа, содержит две произвольные функции одной переменной, удовлетворяющие дифференциальному условию. Однако когда система гидродинамического типа принадлежит к рассматриваемому нами классу, эти произвольные функции принимают конкретную форму одного из трех мономов, так что имеются только три независимых рекурсионных оператора для данной системы. Рассматриваемый класс является достаточно общим и содержит все известные нам примеры.

Рассматриваемые системы связаны с обобщенным линейным волновым уравнением Эйлера-Пуассона-Дарбу (ЭПД): для каждого такого волнового уравнения строится семейство коммутирующих систем гидродинамического типа и соответствующая тройка рекурсионных операторов. Конкретные системы отвечают частным решениям этих волновых уравнений. Легко построить лестничные операторы этих волновых уравнений, с помошью которых оказывается возможным породить иерархии связанных решений. Соответствуюшие системы гидродинамического типа связаны посредством

* Department of Applied Mathematics and Centre for Nonlinear Studies, University of Leeds, UK. E-mail: allan@amsta.leeds.ac.uk, gurel@amsta.leeds.ac.uk

${ }^{\dagger}$ Department of Mathematics, Bilkent University, Ankara,Turkey.

E-mail: gurel@fen.bilkent.edu.tr 
рекурсионных операторов типа оператора Тешукова. Их можно использовать для порождения "высших симметрий”, действуя на некоторые простые симметрии негидродинамического типа.

Для упрощения изложения бо́льшая часть настояшей работы посвящена двумерным системам, однако наши формулы легко обобщаются и на высшие размерности (см. раздел 5).

\section{2. КЛАСС ДВУМЕРНЫХ СИСТЕМ}

В размерности $d=2$ каждая система гидродинамического типа может быть приведена к диагональному виду, так что без потери обшности мы начнем рассмотрение с диагональной системы

$$
q_{t}^{i}=v^{i}(\mathbf{q}) q_{x}^{i}, \quad i=1,2 .
$$

С помощью функций $v^{i}$ можно обычным образом [2] определить диагональную метрику с ковариантными компонентами $g_{i j}$ :

$$
\frac{\partial_{j} v^{i}}{v^{j}-v^{i}}=\frac{1}{2} \partial_{j} \ln g_{i i}, \quad i \neq j
$$

где $\partial_{j} \equiv \partial / \partial q^{j}$.

Все известные нам примеры удовлетворяют условиям

$$
\partial_{2}\left(v^{1} \varphi^{1}\right)=\partial_{1}\left(v^{2} \varphi^{2}\right)
$$

где каждая функция $\varphi^{i}=\varphi^{i}\left(q^{i}\right)$ является функцией одной переменной. Это эквивалентно условию

$$
\partial_{2}\left(\varphi^{1} \ln g_{11}\right)+\partial_{1}\left(\varphi^{2} \ln g_{22}\right)=0 .
$$

Отсюда следует, что существуют функции $V\left(q^{1}, q^{2}\right)$ и $G\left(q^{1}, q^{2}\right)$ такие, что

$$
\begin{aligned}
v^{1} & =\frac{\partial_{1} V}{\varphi^{1}}, & v^{2} & =\frac{\partial_{2} V}{\varphi^{2}} \\
\ln g_{11} & =\frac{\partial_{1} G}{\varphi^{1}}, & \ln g_{22} & =-\frac{\partial_{2} G}{\varphi^{2}} .
\end{aligned}
$$

Эти функции связаны друг с другом соотношением

$$
K(\mathbf{q}) \partial_{1} \partial_{2} V+\frac{1}{\varphi^{1}} \partial_{1} V-\frac{1}{\varphi^{2}} \partial_{2} V=0
$$

где $K(\mathbf{q})=2 / \partial_{1} \partial_{2} G$. (Заметим, что вырожденный случай $\partial_{1} \partial_{2} G=0$ исключается, поскольку для него имеет место равенство $\partial_{2} v^{1}=\partial_{1} v^{2}=0$, в силу которого уравнения (1) становятся несвязанными.) Таким образом, мы имеем линейное волновое уравнение (7) на функцию $V$ с коэффициентами, зависящими от трех функций $\varphi^{i}\left(q^{i}\right)$ и $G\left(q^{1}, q^{2}\right)$. При конкретных значениях коэффициентов это уравнение возникает во многих разделах математики, например в теории сепарабельных гамильтоновых систем [6]. 
В дальнейшем мы ограничим вид этих функций следуюшим образом:

1) выберем $\varphi^{i}$ в виде

$$
\varphi^{i}\left(q^{i}\right)=\frac{1}{\alpha_{i}\left(q^{i}\right)^{n}}, \quad \alpha_{i} \in \mathbb{R}
$$

2) потребуем, чтобы волновое уравнение (7) имело инвариантный вид при преобразовании

$$
\bar{V}\left(\bar{q}^{1}, \bar{q}^{2}\right)=V\left(q^{1}, q^{2}\right)\left(q^{1}\right)^{\alpha_{2}}\left(q^{2}\right)^{\alpha_{1}}, \quad \bar{q}^{i}=f_{i}\left(q^{i}\right) .
$$

Тогда мы сразу находим точный вид $K(\mathbf{q})$ :

$$
K_{n}\left(q^{1}, q^{2}\right)=q^{1} q^{2}\left(\left(q^{1}\right)^{n-1}-\left(q^{2}\right)^{n-1}\right),
$$

где $n \neq 1$ соответствует степени $q^{i}$ в равенстве (8). Оставшиеся условия инвариантности дают при $n \neq 1$

$$
f_{i}\left(q^{i}\right) \equiv \bar{q}^{i}=\frac{1}{q^{i}}, \quad i=1,2 .
$$

Выполнение этих двух ограничений приводит к линейному волновому уравнению

$$
L_{n} V \equiv q^{1} q^{2}\left(\left(q^{1}\right)^{n-1}-\left(q^{2}\right)^{n-1}\right) \partial_{1} \partial_{2} V+\alpha_{1}\left(q^{1}\right)^{n} \partial_{1} V-\alpha_{2}\left(q^{2}\right)^{n} \partial_{2} V=0
$$

которое можно считать обобщением линейного волнового уравнения ЭПД [7] (соответствуюшего $n=0$ и $\alpha_{1}=\alpha_{2}$ ). Некоторые свойства волновых уравнений ЭПД приводятся в приложении.

ЗАмЕчАниЕ. Поскольку $V=1$ - тривиальное решение уравнения (12), то легко построить нетривиальное решение

$$
V=\left(q^{1}\right)^{-\alpha_{2}}\left(q^{2}\right)^{-\alpha_{1}},
$$

исходя из которого можно записать $v^{i}$ согласно условиям (5).

Формула (2) позволяет получить компоненты метрики $g_{i j}$ для всей иерархии, если взять любую выбранную функцию $v^{i}$ (как, например, в (13)) или подставить (5) в (2), тогда с учетом (8) и используя (12), имеем

$$
\partial_{i} \ln g_{j j}=\frac{2 \alpha_{j}\left(q^{j}\right)^{n-1}}{q^{i}\left(\left(q^{j}\right)^{n-1}-\left(q^{i}\right)^{n-1}\right)},
$$

откуда получаем

$$
g_{11}=\frac{\left(q^{2}\right)^{\frac{2 \alpha_{1} n}{n-1}} \gamma^{1}\left(q^{1}\right)}{\left(\left(q^{1}\right)^{n} q^{2}-\left(q^{2}\right)^{n} q^{1}\right)^{\frac{2 \alpha_{1}}{n-1}}}, \quad g_{22}=\frac{\left(q^{1}\right)^{\frac{2 \alpha_{2} n}{n-1}} \gamma^{2}\left(q^{2}\right)}{\left(\left(q^{2}\right)^{n} q^{1}-\left(q^{1}\right)^{n} q^{2}\right)^{\frac{2 \alpha_{2}-1}{n-1}} .}
$$




\section{3. ЛЕСТНИЧНЫЕ И РЕКУРСИОННЫЕ ОПЕРАТОРЫ}

Рассмотрим теперь операторы, действие которых на решение линейного дифференциального уравнения в частных производных порождает другое (возможно, совпадающее с исходным) решение. Это операторный аналог понятия симметрии, называемый лестничным оператором в квантовой механике и в теории специальных функций и рекурсионным оператором в теории интегрируемых уравнений [8]. В последнем случае мы ищем "коммутирующие потоки" ("обобщенные симметрии") нелинейного уравнения. Симметрии удовлетворяют “линеаризованному уравнению”, что и порождает в этом случае соответствующий линейный оператор. Мы пользуемся здесь термином “лестничньй оператор" по отношению к уравнению ЭПД и рекурсионному оператору для систем гидродинамического типа. Мы используем соотношения (5), связываюшие (12) и (1), чтобы построить рекурсионные операторы для системы (1), соответствующие лестничным операторам для уравнения (12).

Пусть $L$ - некоторый линейный дифференциальный оператор в частных производных, а $R$ - некоторый (вообще говоря, интегродифференциальный) оператор, являющийся лестничным или рекурсионным оператором, т.е. он должен удовлетворять условию

$$
[R, L]=k\left(q^{1}, q^{2}\right) L
$$

где $[\cdot, \cdot]$ - коммутатор, а $k\left(q^{1}, q^{2}\right)$ - некоторая функция, определяемая этим соотношением. Условие (15) гарантирует, что если $L f=0$, то $L(R f)=0$.

3.1. Лестничный оператор ЭПД. Рассмотрим теперь обобщенное волновое уравнение ЭПД (12) и построим соответствующие лестничные операторы в виде

$$
r=\xi^{1} \partial_{1}+\xi^{2} \partial_{2}+\xi^{0}
$$

где $\xi^{i}=\xi^{i}\left(q^{1}, q^{2}\right), i=0,1,2$, удовлетворяют условиям (15) с $L_{n}$.

ЛЕмма. Имеются ровно три нетривиальных оператора вида (16), удовлетворяющих условию (15), причем они имеют вид

$$
\begin{aligned}
& r_{1}=\left(q^{1}\right)^{2-n} \partial_{1}+\left(q^{2}\right)^{2-n} \partial_{2}+\alpha_{2}\left(q^{1}\right)^{1-n}+\alpha_{1}\left(q^{2}\right)^{1-n} \\
& r_{2}=q^{1} \partial_{1}+q^{2} \partial_{2} \\
& r_{3}=\left(q^{1}\right)^{n} \partial_{1}+\left(q^{2}\right)^{n} \partial_{2}
\end{aligned}
$$

с точностью до умножсени на константу или прибавления константы.

Используя эту свободу в умножении и сложении, при $n \neq 1$ мы можем преобразовать коммутационные соотношения операторов $r_{i}$ к виду соотношений для стандартного базиса в алгебре $s l(2, \mathbb{C})$ :

$$
r_{+}=\frac{1}{n-1} r_{1}, \quad r_{0}=\frac{1}{n-1} r_{2}+\frac{\alpha_{1}+\alpha_{2}}{2(n-1)}, \quad r_{-}=\frac{1}{n-1} r_{3} .
$$


Операторы $r_{+}, r_{0}, r_{-}$удовлетворяют коммутационным соотношениям

$$
\left[r_{+}, r_{0}\right]=r_{+}, \quad\left[r_{0}, r_{-}\right]=r_{-}, \quad\left[r_{+}, r_{-}\right]=2 r_{0}
$$

Оператор Казимира этой алгебры

$$
\mathcal{C}_{n}=r_{+} r_{-}+r_{-} r_{+}-2 r_{0}^{2}
$$

в явном виде дается формулой (напомним, что $n \neq 1$ )

$$
\mathcal{C}_{n}=\frac{2}{(n-1)^{2}}\left(\left(q^{2}\right)^{1-n}-\left(q^{1}\right)^{1-n}\right) L_{n}-\left(n-1+\frac{\alpha_{1}+\alpha_{2}}{2}\right) \frac{\alpha_{1}+\alpha_{2}}{(n-1)^{2}}
$$

3.2. Гидродинамический рекурсионный оператор. Теперь используем соотношение (5) для построения рекурсионного оператора для системы $(1)$, соответствующего каждому из лестничных операторов (17). Наши вычисления полностью алгебраические, так что выписать их не составляет труда. Один из рекурсионных операторов является нелокальным, но и он вычисляется алгебраическим способом. Более того, лестничньй оператор (16) является скалярным, тогда как соответствующий гидродинамический рекурсионньй оператор - матричный. Очевидно, что гораздо легче построить матричный объект из известного скалярного объекта, нежели вычислить матричный объект исходя из основного определения (15).

Преж де всего напомним, что обший лестничный оператор для волнового уравнения (12) имеет вид (16). Используя соотношения (5), мы приходим к диаграмме

$$
\begin{aligned}
& \begin{array}{cc}
V \stackrel{\frac{q_{x}^{j}}{\varphi^{j}} \partial_{j}}{\longrightarrow} & v_{j} q_{x}^{j} \\
r \downarrow & \downarrow \mathcal{R}_{j}^{i},
\end{array} \\
& r V \stackrel{\frac{q_{x}^{i}}{\varphi^{i}} \partial_{i}}{\longrightarrow} \mathcal{R}_{j}^{i}\left(v_{j} q_{x}^{j}\right)
\end{aligned}
$$

которая коммутативна, только если $\mathcal{R}$ - рекурсионный оператор. Чтобы найти компоненты $\mathcal{R}_{j}^{i}$, нам просто следует решить алгебраическую систему уравнений

$$
\sum_{j=1}^{2} \mathcal{R}_{j}^{i}\left(\frac{q_{x}^{j}}{\varphi^{j}} \partial_{j} V\right)=\frac{q_{x}^{i}}{\varphi^{i}} \partial_{i}(r V), \quad i=1,2
$$

При решении системы (19) следует использовать уравнение (12) для исключения смешанных производных $V$. Мы получаем, что рекурсионные операторы должны быть дифференциальными операторами первого порядка и при $\partial_{i} \xi^{0} \neq 0$ необходимо включить нелокальньй член. 
При общих $r$ и $\varphi^{i}$ решение уравнений (19) дает следующий класс рекурсионных операторов:

$$
\begin{aligned}
& \mathcal{R}_{1}^{1}=\xi^{1} D_{x} \frac{1}{q_{x}^{1}}+\xi^{1} \frac{\partial_{1} \varphi^{1}}{\varphi^{1}}+\frac{\xi^{1} q_{x}^{2}-\xi^{2} q_{x}^{1}}{q_{x}^{1} K_{n} \varphi^{1}}+\partial_{1} \xi^{1}+\xi^{0}+\partial_{1} \xi^{0} \frac{q_{x}^{1}}{\varphi^{1}} D_{x}^{-1} \varphi^{1}, \\
& \mathcal{R}_{2}^{1}=\frac{\xi^{2} q_{x}^{1}-\xi^{1} q_{x}^{2}}{q_{x}^{2} K_{n} \varphi^{1}}+\partial_{1} \xi^{0} \frac{q_{x}^{1}}{\varphi^{1}} D_{x}^{-1} \varphi^{2} \\
& \mathcal{R}_{1}^{2}=\frac{\xi^{2} q_{x}^{1}-\xi^{1} q_{x}^{2}}{q_{x}^{1} K_{n} \varphi^{2}}+\partial_{2} \xi^{0} \frac{q_{x}^{2}}{\varphi^{2}} D_{x}^{-1} \varphi^{1}, \\
& \mathcal{R}_{2}^{2}=\xi^{2} D_{x} \frac{1}{q_{x}^{2}}+\xi^{2} \frac{\partial_{2} \varphi^{2}}{\varphi^{2}}+\frac{\xi^{1} q_{x}^{2}-\xi^{2} q_{x}^{1}}{q_{x}^{2} K_{n} \varphi^{2}}+\partial_{2} \xi^{2}+\xi^{0}+\partial_{2} \xi^{0} \frac{q_{x}^{2}}{\varphi^{2}} D_{x}^{-1} \varphi^{2} .
\end{aligned}
$$

Как было показано выше (см. п. 3.1), обобшенное волновое уравнение ЭПД (12) допускает только три лестничных оператора $r_{-}, r_{0}, r_{+}$, что порождает три независимых функции $\xi^{i}$ (при данном $n \neq 1$ ), которые в свою очередь приводят к трехпараметрическому семейству рекурсионных операторов $\left\{\mathcal{R}_{-}, \mathcal{R}_{0}, \mathcal{R}_{+}\right\}$. Эти операторы удовлетворяют уравнению (15), где матричный линейный дифференциальный оператор $L$ определяется правой частью формулы

$$
\eta_{t}^{i}=v^{i} \eta_{x}^{i}+\sum_{j} q_{x}^{i} \frac{\partial v^{i}}{\partial q^{j}} \eta^{j}
$$

Здесь $\eta^{i}$ представляют компоненты симметрии

$$
q_{\tau}^{i}=\eta^{i}\left(\mathbf{q}, \mathbf{q}_{x}, \ldots\right)
$$

не обязательно гидродинамического типа. Операторы $\mathcal{R}_{-}$и $\mathcal{R}_{0}$ являются чисто дифференциальными, однако $\mathcal{R}_{+}$имеет ненулевой нелокальный член. Фиксация $n$ (и параметров $\alpha_{i}$ ) означает фиксацию гидродинамической иерархии, а не одной системы. Не следует также забывать, что нетривиальное решение

$$
V=\left(q^{1}\right)^{-\alpha_{2}}\left(q^{2}\right)^{-\alpha_{1}}
$$

построенное из тривиального $V=1$, не зависит от $n$.

3.3. Рекурсионные операторы Шефтеля-Тешукова. Рекурсионные операторы для диагональных систем гидродинамического типа исследовались Шефтелем [3, 5] и Тешуковым [4], которые рассматривали рекурсионные операторы вида $A_{1} D_{x}+A_{0}$ для функций $A_{i}$ от $\mathbf{q}, \mathbf{q}_{x}, \ldots$ Все эти операторы вычислены путем непосредственного решения уравнения для рекурсионного оператора. Тешуков показал, что $n$-мерная полугамильтонова система гидродинамического типа допускает рекурсионный оператор вида

$$
R_{j}^{i}=\left(\delta_{j}^{i} C^{i} D_{x}+\Gamma_{i j}^{i}\left(q_{x}^{i} C^{j}-q_{x}^{j} C^{i}\right)+\delta_{j}^{i} C^{j} \sum_{k} q_{x}^{k} \Gamma_{i k}^{i}\right) \frac{1}{q_{x}^{j}}
$$


где $\Gamma_{i j}^{i}=(1 / 2)\left(\partial_{j} \ln g_{i i}\right)$ при $i \neq j-$ коэффициенты связности Кристоффеля некоторой метрики, а каждая функция $C^{i}=C^{i}\left(q^{i}\right)$ является функцией одной переменной. На $R_{j}^{i}$ следует наложить условие, которое можно получить, потребовав, чтобы оператор (21) удовлетворял уравнению для рекурсионного оператора. Это эквивалентно требованию, чтобы оператор (21) отображал систему (1) на коммутирующий гидродинамический поток. В результате действия на систему (1) оператора (21) получаем другую диагональную систему гидродинамического типа $q_{t}^{i}=w^{i} q_{x}^{i}$, в которой

$$
w^{i}=\sum_{j} C^{j}\left(\partial_{j} v^{i}+\Gamma_{i j}^{i} v^{i}\right) .
$$

Диагональность следует из выражения для $\Gamma_{i j}^{i}$ через $v^{i}$. Две системы гидродинамического типа $q_{t_{1}}^{i}=v^{i} q_{x}^{i}$ и $q_{t_{2}}^{i}=w^{i} q_{x}^{i}$ при $i=1,2, \ldots, k$ являются симметриями друг друга тогда и только тогда, когда

$$
\frac{\partial_{j} v^{i}}{v^{j}-v^{i}}=\frac{\partial_{j} w^{i}}{w^{j}-w^{i}}, \quad i \neq j, \quad i, j=1,2, \ldots, k .
$$

Для метрики (14) это условие (при $k=2$ ) приводит к следуюшим условиям на $C^{i}\left(q^{i}\right)$ и $\gamma^{i}$ :

$$
\begin{aligned}
& \left(\dot{C}^{1}+\dot{C}^{2}\right) q^{1} q^{2}\left(\left(q^{1}\right)^{n-1}-\left(q^{2}\right)^{n-1}\right)+n\left(C^{2} q^{1}-C^{1} q^{2}\right)\left(\left(q^{1}\right)^{n-1}+\left(q^{2}\right)^{n-1}\right)+ \\
& \quad+2 C^{1}\left(q^{2}\right)^{n}-2 C^{2}\left(q^{1}\right)^{n}=0 \\
& (n-1)\left(n C^{1} q^{2}-n C^{2} q^{1}+q^{1} q^{2}\left(\dot{C}^{2}-\dot{C}^{1}+C^{1} \frac{\dot{\gamma}^{1}}{\gamma^{1}}-C^{2} \frac{\dot{\gamma}^{2}}{\gamma^{2}}\right)\right)\left(\left(q^{1}\right)^{n-1}-\left(q^{2}\right)^{n-1}\right)+ \\
& \quad+2 \alpha_{2} C^{2} q^{1}\left(\left(q^{1}\right)^{n-1}-n\left(q^{2}\right)^{n-1}\right)+2 \alpha_{1} C^{1} q^{2}\left(\left(q^{2}\right)^{n-1}-n\left(q^{1}\right)^{n-1}\right)+ \\
& \quad+2 \alpha_{1}(n-1) C^{2}\left(q^{1}\right)^{n}+2 \alpha_{2}(n-1) C^{1}\left(q^{2}\right)^{n}=0
\end{aligned}
$$

где $\dot{C}^{i}, \dot{\gamma}^{i}$-производные по аргументу функции. Уравнение (23) несложно решить. Дифференцируя его $n+1$ раз по $q^{1}$ или $q^{2}$, получим уравнения только на одну из двух функций $C^{i}\left(q^{i}\right)$. Мы получаем, что $C^{1}$ и $C^{2}$ являются одинаковыми функциями, но разных аргументов:

$$
C^{i}=S\left(q^{i}\right), \quad S(x)=\beta_{0} x^{n}+\beta_{1} x+\beta_{2} x^{2-n}, \quad n \neq 1 .
$$

Подставляя эти выражения для $C^{i}$ в $(24)$, получаем уравнения для метрических функций $\gamma^{i}$, которые имеют следуюшие решения:

1) если $C^{i}=\left(q^{i}\right)^{n}$, то $\gamma^{i}=\beta_{i}\left(q^{i}\right)^{2 \alpha_{i} n /(n-1)}, i=1,2$;

2) если $C^{i}=\left(q^{i}\right)^{2-n}$, то $\gamma^{i}=\beta_{i}\left(q^{i}\right)^{2\left(\alpha_{i}+(n-1) \alpha_{j}-(n-1)^{2}\right) /(n-1)}$ при $j \neq i=1,2$;

3) если $C^{i}=q^{i}$, то $\gamma^{i}=\beta_{i}\left(q^{i}\right)^{\left(2 \alpha_{i}+\lambda_{i}\right) /(n-1)}, i=1,2$,

где $\beta_{i}$ и $\lambda_{i}$-произвольные константы. Этот результат доказьвает, что для рассматриваемого класса имеются ровно три рекурсионных оператора типа оператора Шефтеля-Тешукова. Два из них совпадают (с точностью до аддитивной постоянной), если 
строить их с помощью лестничных операторов уравнений ЭПД (при $C^{i}=\xi^{i}$ и для выбранных $C^{i}$ и $\gamma^{i}$ ). Операторы Шефтеля-Тешукова содержат $\gamma^{i}$ в члене $\Gamma_{i i}^{i}$, а полученные нами операторы не содержат. Рекурсионные операторы (20) применимы для функций $\xi^{i}$ тех же трех видов, но для произвольных $\gamma^{i}$. Это, однако, не имеет последствий для систем гидродинамического типа, поскольку они не зависят от $\gamma^{i}$. Найденный нами нелокальный рекурсионный оператор не принадлежит к классу Шеффтеля-Тешукова.

3.4. Иерархии симметрий. По построению рекурсионные операторы (20) приводят к диагональным гидродинамическим симметриям при действии на диагональные системы гидродинамического типа. Действие оператора $\mathcal{R}$ на систему (1) обшего вида дается формулой

$$
\sum_{j} \mathcal{R}_{j}^{i} v^{j} q_{x}^{j}=\left[\sum_{j} \xi^{j} \partial_{j} v^{i}+\left(\partial_{i} \xi^{i}+\xi^{0}+\xi^{i} \frac{\partial_{i} \varphi^{i}}{\varphi^{i}}\right) v^{i}+\frac{\partial_{i} \xi^{0}}{\varphi^{i}} V\right] q_{x}^{i}
$$

где $V$ - решение уравнения (12), соответствуюшее $v^{i}$. Это в точности соответствует обходу по трем сторонам коммутативной диаграммы (18).

Можно также построить высшие симметрии исходя из некоторой симметрии негидродинамического типа. Например, при выборе $v^{i}$ в виде однородной функции от $q^{1}$ и $q^{2}$ система (1) допускает масштабную симметрию, которую можно записать в эволюционном виде как

$$
q_{t_{s}}^{i}=x q_{x}^{i}+a t q_{t}^{i}-b q^{i}=x q_{x}^{i}+a t v^{i} q_{x}^{i}-b q^{i}
$$

где постоянные $a$ и $b$ - соответственно веса́ $t$ и $q^{i}$ относительно масштабных преобразований. Если $v^{i}$ - однородная функция степени $m$, то ее вес относительно масштабных преобразований равен $m b$. Для баланса весов двух частей уравнения (1) должно выполняться условие $a=1-m b$. В результате действия на уравнение (27) любым из наших рекурсионных операторов мы получаем симметрии второго порядка системы (1). Поскольку член $q_{t}^{i}$ имеет гидродинамический тип, он непосредственно отображается на одну из гидродинамических симметрий, что дает дополнительное слагаемое в окончательной формуле. Нелокальный член в операторе $\mathcal{R}_{+}$мог бы породить нелокальные симметрии, но этого можно избежать, выбирая $b=1 /(n-1)$. Получаюшиеся высшие симметрии, вообще говоря, явно зависят от $x$. Когда функция $\varphi^{i}$ имеет вид (8), применение рекурсионного оператора (20) к уравнению $(27)$ при $b=1 /(n-1)$ дает

$$
\begin{aligned}
q_{\tau}^{i}= & \sum_{j} \mathcal{R}_{j}^{i} q_{t_{s}}^{j}=\frac{\xi^{i} q^{i} q_{x x}^{i}}{(n-1)\left(q_{x}^{i}\right)^{2}}+2 \xi^{i}+\left(\partial_{i} \xi^{i}+\xi^{0}\right) \frac{q^{i}}{1-n}+ \\
& +\frac{\alpha_{i}\left(q^{i}\right)^{n}}{(1-n) K_{n}}\left(\frac{\xi^{i} q^{i} q_{x}^{j}}{q_{x}^{i}}+\frac{\xi^{j} q^{j} q_{x}^{i}}{q_{x}^{j}}-\left(\xi^{i} q^{j}+\xi^{j} q^{i}\right)\right)+a t w^{i} q_{x}^{i}+ \\
& +\left(\frac{-n \xi^{i}}{q^{i}}+\partial_{i} \xi^{i}+\xi^{0}+\frac{\partial_{i} \xi^{0} q^{i}}{1-n}+\frac{\alpha_{i} \partial_{i} \xi^{0}}{\alpha_{j}(1-n)}\left(q^{i}\right)^{n}\left(q^{j}\right)^{1-n}\right) x q_{x}^{i}
\end{aligned}
$$

где $w^{i}$ - выражение в квадратных скобках формулы (26). 
Ни операторы Шефтеля-Тешукова, ни наши рекурсионные операторы не обладают наследственными свойствами, так что алгебра симметрий, вообше говоря, неабелева. В то время как гидродинамические симметрии взаимно коммутируют, симметрии высшего порядка, вообше говоря, нет.

\section{4. ПРИМЕРЫ В РАЗМЕРНОСТИ $d=2$}

Рассмотрим некоторые примеры систем гидродинамического типа из нашего класса. В простейших случаях имеем $n=0$ и $\alpha_{1}=\alpha_{2}=\alpha$. Даже в этом подклассе имеется много хорошо известных примеров. Мы не будем явно строить рекурсионные операторы, поскольку они выглядят достаточно сложно (хотя их и легко получить подстановкой B $(20))$.

4.1. Случай $n=0$. При $\alpha_{1}=\alpha_{2}=\alpha$ уравнение (12) является обычным линейным волновым уравнением ЭПД

$$
\left(q^{2}-q^{1}\right) \partial_{1} \partial_{2} V+\alpha\left(\partial_{1} V-\partial_{2} V\right)=0
$$

Известно, что уравнение (29) имеет бесконечно много однородных полиномиальных решений [7] (см. также приложение). Каждое решение при фиксированном $\alpha$ соответствует определенному члену в гидродинамической иерархии, а каждый член является симметрией для других (удовлетворяющих условию (22)).

Эти наблюдения гарантируют, что для построения рекурсионных операторов достаточно найти решения, порождаюшие простейшие системы гидродинамического типа. Пусть $V_{N}(\alpha)=V_{N}\left(q^{1}, q^{2} ; \alpha\right)$ - однородное полиномиальное решение (или решения) (29) $N$-го порядка. Некоторые из этих решений имеют вид

$$
\begin{aligned}
& V_{1}(\alpha)=q^{1}+q^{2}, \\
& V_{2}(\alpha)= \begin{cases}\left(q^{1}\right)^{2}+\frac{2 \alpha}{\alpha+1} q^{1} q^{2}+\left(q^{2}\right)^{2}, & \alpha \neq-1, \\
q^{1} q^{2}, & \alpha=-1,\end{cases} \\
& V_{3}(\alpha)= \begin{cases}\left(q^{1}\right)^{3}+\frac{3 \alpha}{\alpha+2}\left(\left(q^{1}\right)^{2} q^{2}+q^{1}\left(q^{2}\right)^{2}\right)+\left(q^{2}\right)^{3}, & \alpha \neq-2, \\
\left(q^{1}\right)^{2} q^{2}+q^{1}\left(q^{2}\right)^{2}, & \alpha=-2 .\end{cases}
\end{aligned}
$$

Решение $V_{1}(\alpha)$ не порождает нетривиальной системы гидродинамического типа, поскольку соответствующие функции $v^{i}$ являются постоянными, что приводит к тому, что уравнения системы (1) становятся несвязанными. Наоборот, решение $V_{2}(\alpha)$ порождает бесконечное семейство, содержашее некоторые известные примеры. Используя соотношения (5), можно найти $v^{i}$ для $V_{2}(\alpha)$ (опуская несушественный постоянный множитель) в виде

$$
\begin{gathered}
v^{1}(\alpha)=\partial_{1} V_{2}(\alpha)=q^{1}+\frac{\alpha}{\alpha+1} q^{2}, \quad v^{2}(\alpha)=\partial_{2} V_{2}(\alpha)=q^{2}+\frac{\alpha}{\alpha+1} q^{1}, \quad \alpha \neq-1, \\
v^{1}=q^{2}, \quad v^{2}=q^{1}, \quad \alpha=-1 .
\end{gathered}
$$


Система гидродинамического типа, определенная из (33), лежит в классе так называемых линейно вырожденных систем и хорошо изучена $[9,10]$. Чтобы продемонстрировать ее симметрийные свойства, рассмотрим решение $V_{3}(-1)$ и запишем соответствующие $w^{i}$ (используя (5)):

$$
w^{1}=\left(q^{1}\right)^{2}-2 q^{1} q^{2}-\left(q^{2}\right)^{2}, \quad w^{2}=\left(q^{2}\right)^{2}-2 q^{1} q^{2}-\left(q^{1}\right)^{2} .
$$

Поскольку $v^{i}$ и $w^{i}$ удовлетворяют тождеству (22), соответствующие системы гидродинамического типа коммутируют. Другие известные примеры из этого класса представляют собой случаи, когда $\alpha= \pm 1 / 2$. При $\alpha=1 / 2$ мы имеем систему

$$
q_{t}^{1}=\left(3 q^{1}+q^{2}\right) q_{x}^{1}, \quad q_{t}^{2}=\left(3 q^{2}+q^{1}\right) q_{x}^{2}
$$

описываюшую волны в мелкой воде (специальный случай квазиклассического предела связанных уравнений Кортевега-де Фриза (Кд $\Phi)$, изучавшихся в [11]). При $\alpha=-1 / 2$ получаем систему

$$
q_{t}^{1}=\left(q^{1}-q^{2}\right) q_{x}^{1}, \quad q_{t}^{2}=\left(q^{2}-q^{1}\right) q_{x}^{2}
$$

которая простым образом связана с бездисперсионной иерархией Тоды, обсуждавшейся в [12].

4.2. Случай $n=2$. Этот случай несколько сложнее, чем случай $n=0$, отчасти потому, что мы не знаем вида общего полиномиального решения. Тем не менее можно построить примеры систем гидродинамического типа. Например, полагая $\alpha_{1}=\alpha_{2}=-1$, получаем обобшенное волновое уравнение ЭПД

$$
q^{1} q^{2}\left(q^{1}-q^{2}\right) \partial_{1} \partial_{2} V-\left(q^{1}\right)^{2} \partial_{1} V+\left(q^{2}\right)^{2} \partial_{2} V=0 .
$$

Нетривиальное решение этого уравнения можно построить исходя из тривиального решения $V=1$ в виде $V=q^{1} q^{2}$, что дает следуюшие функшии $v^{i}$ :

$$
v^{1}=\left(q^{1}\right)^{2} \partial_{1} V=\left(q^{1}\right)^{2} q^{2}, \quad v^{2}=\left(q^{2}\right)^{2} \partial_{2} V=q^{1}\left(q^{2}\right)^{2},
$$

которые принадлежат классу Темпла [9]. Действие оператором $r_{-}$(или $\mathcal{R}_{-}$) дает

$$
w^{1}=\left(q^{1}\right)^{2} q^{2}\left(2 q^{1}+q^{2}\right), \quad w^{2}=q^{1}\left(q^{2}\right)^{2}\left(q^{1}+2 q^{2}\right) .
$$




\section{N-КОМПОНЕНТНЫЕ СИСТЕМЫ}

Легко обобшить полученные результаты на системы гидродинамического типа в размерности $d=N$ :

$$
q_{t}^{i}=v^{i}(\mathbf{q}) q_{x}^{i}, \quad i=1,2, \ldots, N .
$$

В размерности $d=N$ известны примеры, которые удовлетворяют уравнению, аналогичному (4),

$$
\partial_{j}\left(v^{i} \varphi^{i}\right)=\partial_{i}\left(v^{j} \varphi^{j}\right), \quad i \neq j,
$$

позволяющему получить следующие условия на метрику:

$$
\partial_{j}\left(\varphi^{i} \ln g_{i i}\right)+\partial_{i}\left(\varphi^{j} \ln g_{j j}\right)=0 .
$$

Из соотношения (35) следует сушествование функции $V(\mathbf{q})$, что дает

$$
v^{i}=\frac{\partial_{i} V}{\varphi^{i}} \quad \forall i=1,2, \ldots, N .
$$

Используя (2), находим многокомпонентные обобщенные уравнения ЭПД для функции $V$

$$
K^{i j} \partial_{i} \partial_{j} V+\frac{\partial_{i} V}{\varphi^{i}}-\frac{\partial_{j} V}{\varphi^{j}}=0 \quad \forall i \neq j
$$

где $K^{i j}(\mathbf{q})=2 /\left(\varphi^{i} \partial_{j} \ln g_{i i}\right)$ и

$$
g_{i i}=\gamma^{i}\left(q^{i}\right) \prod_{j \neq i} \frac{\left(q^{j}\right)^{\frac{2 \alpha_{i} \alpha_{i j} n}{n-1}}}{\left(\left(q^{i}\right)^{n} q^{j}-\left(q^{j}\right)^{n} q^{i}\right)^{\frac{2 \alpha_{i} \alpha_{i j}}{n-1}}} .
$$

Отметим, что существует $(1 / 2) N(N-1)$ таких уравнений. Определение (8) остается без изменений, однако условие инвариантности (9) принимает вид

$$
\bar{V}(\mathbf{q})=V(\mathbf{q}) \prod_{k=1}^{N}\left(q^{k}\right)^{\alpha_{k}}, \quad \bar{q}^{i}=f^{i}\left(q^{i}\right),
$$

где $\alpha_{k}=\prod_{j \neq k} \alpha_{j}$. Вследствие условия инвариантности получаем $(1 / 2) N(N-1)$ уравнений

$$
L_{n}^{i j} V \equiv \frac{1}{\alpha_{i j}} q^{i} q^{j}\left(\left(q^{i}\right)^{n-1}-\left(q^{j}\right)^{n-1}\right) \partial_{i} \partial_{j} V+\alpha_{i}\left(q^{i}\right)^{n} \partial_{i} V-\alpha_{j}\left(q^{j}\right)^{n} \partial_{j} V=0,
$$

где $\alpha_{i j}=\prod_{k \neq i, j} \alpha_{k}$. Для каждого уравнения в этой системе можно найти лестничные операторы в виде

$$
\begin{aligned}
& r_{1}^{i j}=\left(q^{i}\right)^{2-n} \partial_{i}+\left(q^{j}\right)^{2-n} \partial_{j}+\alpha_{j}\left(q^{i}\right)^{1-n}+\alpha_{i}\left(q^{j}\right)^{1-n}, \\
& r_{2}^{i j}=q^{i} \partial_{i}+q^{j} \partial_{j}, \\
& r_{3}^{i j}=\left(q^{i}\right)^{n} \partial_{i}+\left(q^{j}\right)^{n} \partial_{j}
\end{aligned}
$$

с точностью до умножения на константу или прибавления константы.

Как и ранее, можно построить коммутативную диаграмму и решить алгебраические уравнения на компоненты рекурсионного оператора $\mathcal{R}$; распространение наших предыдуших примеров на $N$-мерный случай является несложным упражнением. 


\section{6. ЗАКЛЮЧЕНИЕ}

Мы показали, как рекурсионные операторы для систем гидродинамического типа могут быть связаны с лестничными операторами обобшенного уравнения ЭПД, которое используется для построения функций $v^{i}$ в уравнении (1) с помощью формул (5). Это отражает сушествование богатого семейства симметрий в рамках полугамильтоновых систем гидродинамического типа. Такое явление контрастирует с обычными солитонными уравнениями, такими как уравнение КдФ, где иерархии симметрий, как правило, дискретны.

В последнее время значительный интерес привлекают к себе неоднородные системы гидродинамического типа, такие как уравнение Гиббона-Царева [13]

$$
q_{t}^{1}=q^{2} q_{x}^{1}+\frac{1}{q^{1}-q^{2}}, \quad q_{t}^{2}=q^{1} q_{x}^{2}+\frac{1}{q^{2}-q^{1}} .
$$

Это уравнение, как представляется, имеет только конечное число симметрий, так что не следует ожидать, что для него сушествует рекурсионный оператор. Теорема Царева [2] о законах сохранения, симметриях и обобшенном преобразовании годографа в этом случае не выполняется, так что такие уравнения и в самом деле дают весьма интересный материал для будущих исследований.

Благодарности. Т. Б. Гюрель хотел бы поблагодарить TUBITAK за грант, позволивший ему посетить Лидс в 1997-1998 академическом году. Он также благодарит университет Лидс и, в частности, сотрудников группы по изучению интегрируемых систем за гостеприимство. Мы благодарим М. Б. Шефтеля за предоставление экземпляров его статей и С. П. Царева за обсуждения и прочтение предварительного варианта рукописи.

ПРИЛОЖЕНИЕ

\section{Уравнения ЭПД}

Укажем некоторые полезные свойства линейных волновых уравнений ЭПД

$$
L_{0} u \equiv(x-y) u_{x y}+\alpha u_{y}-\beta u_{x}=0 .
$$

В случае $\alpha=\beta$ это уравнение выводится из уравнения

$$
u_{r r}+\frac{m}{r} u_{r}-u_{t t}=0
$$

с помощью простого координатного преобразования $x=t+r, y=t-r$ при $m=2 \alpha$ [7].

Общая формула для однородного полиномиального решения уравнения (36) $N$-го порядка имеет вид $u(x, y)=\mathcal{F}_{N}(x, y)$, где

$$
\mathcal{F}_{N}(x, y)=\sum_{i+j=N} \frac{\alpha_{i} \beta_{j}}{i ! j !} x^{i} y^{j}
$$

$\alpha_{i}=\alpha(\alpha+1) \ldots(\alpha+i-1)$ при $i \geqslant 1$ и $\alpha_{0}=1$ с $i, j \in \mathbb{Z}_{+} \cup\{0\}$. Эта формула приведена в работе [7] для редукции к случаю $\alpha=\beta$. 


\section{Список литературы}

[1] Б. А. Дубровин, С. П. Новиков. ДАН СССР. 1983. Т. 270. С. 781-785.

[2] С. П. Царев. Изв. АН СССР. Сер. матем. 1990. Т. 54. № 2. С. 1048-1068.

[3] М. Б. Шефтель. ТМФ. 1983. Т. 56. С. 387-394.

[4] В. М. Тешуков. Гиперболические системы, допускающие нетривиальную группу Ли-Беклунда. Препринт № 106. Л.: ЛИИАН, 1989.

[5] M. B. Sheftel. Generalised hydrodynamic-type systems. In: CRC Handbook of Lie Group Analysis of Differential Equations. Ed. N. H. Ibragimov. New York: CRC Press, 1996. V. 3. № 7. P. 169-189.

[6] E. G. Kalnins, S. Benenti, W. Miller, Jr. J. Math. Phys. 1997. V. 38. P. 2345-2365.

[7] E. T. Copson. Partial Differential Equations. Cambridge: Cambridge Univ. Press, 1975.

[8] П. Олвер. Приложения групп Ли к дифференциальным уравнениям. М.: Мир, 1989.

[9] E. V. Ferapontov. Hydrodynamic-type systems. In: CRC Handbook of Lie Group Analysis of Differential Equations. Ed. N. H. Ibragimov. New York: CRC Press, 1994. V. 1. № 14 . P. 303-331.

[10] E. V. Ferapontov, A. P. Fordy. J. Geom. Phys. 1997. V. 21. P. 169-182.

[11] E. V. Ferapontov, M. V. Pavlov. Physica D. 1991. V. 52. P. 211-219.

[12] D. Fairlie, I. A. B. Strachan. Physica D. 1996. V. 90. P. 1-8.

[13] J. Gibbons, S. P. Tsarev. Phys. Lett. A. 1996. V. 211. P. 19-24. 